\title{
A REMARK ON O'HARA'S ENERGY OF KNOTS
}

\author{
NOBUMITSU NAKAUCHI \\ (Communicated by Frederick R. Cohen)
}

\begin{abstract}
We show a relation between O'Hara's energy of knots and the Douglas functional.
\end{abstract}

In his paper [4], O'Hara introduced the following energy of knots

$$
E_{\mathrm{knot}}(\varphi):=\frac{1}{2} \iint_{S^{1} \times S^{1}}\left\{\frac{1}{|\varphi(x)-\varphi(y)|^{2}}-\frac{\pi^{2}}{\sin ^{2} \pi(x-y)}\right\} d x d y
$$

for any $C^{2}$-embedding $\varphi: S^{1}=\mathbb{R} / \mathbb{Z} \rightarrow \mathbb{R}^{3}$ such that $\left|\varphi^{\prime}\right|=1$. This quantity is admitted to be negative, but it is bounded from below $\left(E_{\mathrm{knot}}(\varphi) \geq-2\right)$ and blows up if a knot has a self-intersection. O'Hara showed that there exist only finitely many ambient isotopy classes of knots whose energy and bending energy (see [4] for the definition) are bounded from above by any given constant.

In his prize winning paper [3] for the Plateau problem, Douglas employed the Douglas functional

$$
\begin{aligned}
D(\varphi) & =\frac{1}{16 \pi} \int_{0}^{2 \pi} \int_{0}^{2 \pi} \frac{\left|\hat{\varphi}\left(e^{\sqrt{-1} \alpha}\right)-\hat{\varphi}\left(e^{\sqrt{-1} \beta}\right)\right|^{2}}{\sin ^{2}(\alpha-\beta / 2)} d \alpha d \beta \\
& =\frac{\pi}{4} \iint_{S^{1} \times S^{1}} \frac{|\varphi(x)-\varphi(y)|^{2}}{\sin ^{2} \pi(x-y)} d x d y,
\end{aligned}
$$

where $\hat{\varphi}\left(e^{\sqrt{-1} \alpha}\right)=\varphi(\alpha / 2 \pi)$. (See also Struwe [7].) The Douglas functional is closely related to the Dirichlet integral (or more generally the energy functional) of a $C^{1}$-map $f: B^{2} \rightarrow \mathbb{R}^{3}$

$$
E(f)=\frac{1}{2} \iint_{B^{2}}\|d f\|=\frac{1}{2} \iint_{B^{2}}\left(\left\|\frac{\partial f}{\partial x}\right\|^{2}+\left\|\frac{\partial f}{\partial y}\right\|^{2}\right) d x d y,
$$

where $B^{2}=\{z=x+\sqrt{-1} y \in \mathbb{C} ;|z|<1\}$. Indeed $D(\varphi)$ is equal to the energy $E\left(f_{\varphi}\right)$ of the harmonic extension $f_{\varphi}$ of $\varphi$, i.e., $\Delta f_{\varphi}=0$ in $B^{2}, f_{\varphi}=\hat{\varphi}$ on $\partial B^{2}$. (See Douglas [3], Ahlfors [1, Theorems 2-5].) Courant [2] minimized the Dirichlet functional to solve the Plateau problem. The Douglas functional of a curve $\varphi$ is an energy of the harmonic surface $f_{\varphi}$ with the boundary $\varphi$. In other

Received by the editors July 24, 1991 and, in revised form, August 23, 1991.

1991 Mathematics Subject Classification. Primary 58E12; Secondary 57M25.

Key words and phrases. Energy, Douglas functional, Plateau problem, knot, embedding. 
words, the Douglas functional is a "tension" of a strained surface spanning the curve $\varphi$. In contrast, O'Hara's energy of a knotted curve presents a "repulsion" between segments of the curve. If the curve shrinks, O'Hara's energy increases while the Douglas functional decreases. In this note, we show the following relation between these two energies.

Theorem. $\left(E_{\mathrm{knot}}(\varphi)+4\right) D(\varphi) \geq 1 / 2 \pi$.

This result does not seem to be sharp, since our proof uses only the Schwarz inequality.

Proof of Theorem. Let $\lambda: S^{1}=\mathbb{R} / \mathbb{Z} \rightarrow \mathbb{R}$ be a function defined by $\lambda(x)=$ $\min \{x, 1-x\} \quad(x \in[0,1])$. Note that $\lambda$ can be regarded as a piecewise linear periodic function on $\mathbb{R}$, which has the same order at the corners as the function $|x|$. Since $\sin \pi x \leq \pi x \quad\left(0 \leq x \leq \frac{1}{2}\right)$, we see

$$
|\sin \pi x| \leq \pi \lambda(x) \text {. }
$$

We claim

$$
|\varphi(x)-\varphi(y)| \leq \lambda(|x-y|) .
$$

Indeed if $|x-y| \leq \frac{1}{2}$, we know $|\varphi(x)-\varphi(y)| \leq|x-y|=\lambda(|x-y|)$, since $\left|\varphi^{\prime}\right|=1$. Suppose $|x-y|>\frac{1}{2}$. Without loss of generality, we may assume $0 \leq x<y<1$. Then $y>x+\frac{1}{2}$; hence, $\lambda(|x-y|)=1-(y-x)$. Since $\varphi$ is periodic on $\mathbb{R}$, we have $|\varphi(x)-\varphi(y)|=|\varphi(x+1)-\varphi(y)| \leq|x+1-y|=\lambda(|x-y|)$.

We see that

$$
\begin{aligned}
\iint_{S^{1} \times S^{1}} & \left(\frac{\pi^{2}}{\sin ^{2} \pi(x-y)}-\frac{1}{\lambda(|x-y|)^{2}}\right) d x d y \\
= & \int_{0}^{1} \int_{y}^{y+1 / 2}\left(\frac{\pi^{2}}{\sin ^{2} \pi(x-y)}-\frac{1}{|x-y|^{2}}\right) d x d y \\
& +\int_{0}^{1} \int_{y+1 / 2}^{y+1}\left(\frac{\pi^{2}}{\sin ^{2} \pi(x-y)}-\frac{1}{(1-|x-y|)^{2}}\right) d x d y \\
= & \int_{0}^{1} \lim _{\varepsilon \downarrow 0} \int_{y+\varepsilon}^{y+1 / 2}\left(\frac{\pi^{2}}{\sin ^{2} \pi(x-y)}-\frac{1}{|x-y|^{2}}\right) d x d y \\
& +\int_{0}^{1} \lim _{\varepsilon \downarrow 0} \int_{y+1 / 2}^{y+1-\varepsilon}\left(\frac{\pi^{2}}{\sin ^{2} \pi(x-y)}-\frac{1}{(1-|x-y|)^{2}}\right) d x d y \\
= & \int_{0}^{1} \lim _{\varepsilon \downarrow 0} \int_{\varepsilon}^{1 / 2}\left(\frac{\pi^{2}}{\sin ^{2} \pi r}-\frac{1}{r^{2}}\right) d r d y \\
& +\int_{0}^{1} \lim _{\varepsilon \downarrow 0} \int_{1 / 2}^{1-\varepsilon}\left(\frac{\pi^{2}}{\sin ^{2} \pi r}-\frac{1}{(1-r)^{2}}\right) d r d y \\
= & 2 \int_{0}^{1} \lim _{\varepsilon \downarrow 0} \int_{\varepsilon}^{1 / 2}\left(\frac{\pi^{2}}{\sin ^{2} \pi r}-\frac{1}{r^{2}}\right) d r d y \\
= & 2 \int_{0}^{1} \lim _{\varepsilon \downarrow 0}\left\{\pi \cot \pi \varepsilon-\frac{1}{\varepsilon}+2\right\} d y=4 .
\end{aligned}
$$


Then we have

$$
\begin{aligned}
E_{\mathrm{knot}}(\varphi)= & \frac{1}{2} \int_{0}^{1} \int_{0}^{1}\left\{\frac{1}{|\varphi(x)-\varphi(y)|^{2}}-\frac{1}{\lambda(|x-y|)^{2}}\right\} d x d y \\
& -\frac{1}{2} \int_{0}^{1} \int_{0}^{1}\left\{\frac{\pi^{2}}{\sin ^{2} \pi(x-y)}-\frac{1}{\lambda(|x-y|)^{2}}\right\} d x d y \\
= & \frac{1}{2} \int_{0}^{1} \int_{0}^{1}\left\{\frac{1}{|\varphi(x)-\varphi(y)|^{2}}-\frac{1}{\lambda(|x-y|)^{2}}\right\} d x d y-2 \\
= & \frac{1}{2} \int_{0}^{1} \int_{0}^{1} \frac{1}{\lambda(|x-y|)^{2}}\left\{\frac{\lambda(|x-y|)^{2}}{|\varphi(x)-\varphi(y)|^{2}}-1\right\} d x d y-2 \\
\geq & 2 \int_{0}^{1} \int_{0}^{1}\left\{\frac{\lambda(|x-y|)^{2}}{|\varphi(x)-\varphi(y)|^{2}}-1\right\} d x d y-2 \quad\left(\because(2) \text { and } \lambda \leq \frac{1}{2}\right) \\
= & 2 \int_{0}^{1} \int_{0}^{1} \frac{\lambda(|x-y|)^{2}}{|\varphi(x)-\varphi(y)|^{2}} d x d y-4, \quad
\end{aligned}
$$

i.e.,

$$
E_{\mathrm{knot}}(\varphi)+4 \geq 2 \int_{0}^{1} \int_{0}^{1} \frac{\lambda(|x-y|)^{2}}{|\varphi(x)-\varphi(y)|^{2}} d x d y
$$

On the other hand, (1) implies that

$$
D(\varphi) \geq \frac{1}{4 \pi} \int_{0}^{1} \int_{0}^{1} \frac{|\varphi(x)-\varphi(y)|^{2}}{\lambda(|x-y|)^{2}} d x d y .
$$

Then, by the Schwarz inequality, we have

$$
\begin{aligned}
& \left(E_{\mathrm{knot}}(\varphi)+4\right) D(\varphi) \\
& \quad \geq 2 \int_{0}^{1} \int_{0}^{1} \frac{\lambda(|x-y|)^{2}}{|\varphi(x)-\varphi(y)|^{2}} d x d y \frac{1}{4 \pi} \int_{0}^{1} \int_{0}^{1} \frac{|\varphi(x)-\varphi(y)|^{2}}{\lambda(|x-y|)^{2}} d x d y \\
& \quad \geq \frac{1}{2 \pi}\left\{\int_{0}^{1} \int_{0}^{1} d x d y\right\}^{2}=\frac{1}{2 \pi} .
\end{aligned}
$$

Remark. Let $\varphi_{0}$ be a standard circle, i.e., $\varphi_{0}(x)=\left(\frac{1}{2 \pi} \cos 2 \pi x, \frac{1}{2 \pi} \sin 2 \pi x, 0\right)$. We see

$$
\begin{aligned}
\left|\varphi_{0}(x)-\varphi_{0}(y)\right|^{2} & =\frac{1}{4 \pi^{2}}\left\{(\cos 2 \pi x-\cos 2 \pi y)^{2}+(\sin 2 \pi x-\sin 2 \pi y)^{2}\right\} \\
& =\frac{1}{4 \pi^{2}}\{2-2(\cos 2 \pi x \cos 2 \pi y+\sin 2 \pi x \sin 2 \pi y)\} \\
& =\frac{1}{2 \pi^{2}}\{1-\cos 2 \pi(x-y)\}=\frac{1}{\pi^{2}} \sin ^{2} \pi(x-y)
\end{aligned}
$$

hence, we know $E_{\mathrm{knot}}\left(\varphi_{0}\right)=0$ and $D\left(\varphi_{0}\right)=\frac{1}{4 \pi}$. Then $\left(E_{\mathrm{knot}}\left(\varphi_{0}\right)+4\right) D\left(\varphi_{0}\right)$ $=\frac{1}{\pi}$.

\section{REFERENCES}

1. L. V. Ahlfors, Conformal invariants, McGraw-Hill, New York, 1973.

2. R. Courant, Dirichlet's principle, conformal mapping and minimal surfaces, Springer, New York, 1977. 
3. J. Douglas, Solution to the problem of Plateau, Trans. Amer. Math. Soc. 33 (1931), 263-321.

4. J. O'Hara, Energy of knot, Topology 30 (1991), 241-247.

5. __ Family of energy functionals of knot, preprint.

6. __ Energy functionals of knots, preprint.

7. M. Struwe, Plateau's problem and the calculus of variations, Princeton Univ. Press, Princeton, NJ, 1988.

Department of Mathematics, Faculty of Science, Yamaguchi University, Yamaguchi 753, JAPAN

E-mail address: e76220@sinet.ad.jp 